\title{
The Predictive Value of Serum Cytokines for Distinguishing Celiac Disease from Non-Celiac Gluten Sensitivity and Healthy Subjects
}

\author{
Fatemeh Masaebi ${ }^{1,}$ Mehdi Azizmohammad Looha ${ }^{1}$, Mohammad Rostami-Nejad ${ }^{2 *}$, \\ Mohamad Amin Pourhoseingholi ${ }^{2}$, Navid Mohseni ${ }^{1}$, Gabriel Samasca ${ }^{3}$, \\ Iulia Lupan ${ }^{4}$, Mostafa Rezaei-Tavirani ${ }^{5}$ and Mohammad Reza Zali ${ }^{2}$
}

\begin{abstract}
${ }^{1}$ Depatment of Biostatistics, Faculty of Paramedical Sciences, Shahid Beheshti University of Medical Sciences, Tehran, Iran; ${ }^{2}$ Gastroenterology and Liver Diseases Research Center, Research Institute for Gastroenterology and Liver Diseases, Shahid Beheshti University of Medical Sciences, Tehran, Iran; ${ }^{3}$ Department of Immunology, Iuliu Hatieganu University of Medicine and Pharmacy ClujNapoca, Romania; ${ }^{4}$ Department of Molecular Biology and Biotechnology, BabesBolyai University, Cluj-Napoca, Romania; ${ }^{5}$ Proteomics Research Center, Faculty of Paramedical Sciences, Shahid Beheshti University of Medical Sciences, Tehran, Iran
\end{abstract}

Received 3 August 2019; accepted 23 November 2019; published online 1 June 2020

\begin{abstract}
Background: It has been established that the level of some inflammatory cytokines increases in CD and NCGS in comparison with healthy subjects. Therefore, the primary interest in our research was proposing an accurate tool to diagnose patients with CD and NCGS from healthy individuals in an Iranian population. Methods: The serum samples were examined in 171 participants, including $110 \mathrm{CD}$ patients, 46 healthy individuals, and 15 NCGS. The commercial ELISA kits were used to detect the level of the following cytokines: IL-1, IL-6, IL-8, IL-15, and IFN- $p$. The ROC curve analysis was applied to determine the optimal thresholds for high sensitivity, specificity, positive and negative predictive values of cytokines, as the indicators of CD, NCGS, and healthy control groups. Results: In NCGS group, the values of AUC for IL-1, IL-8, and IFN- $\gamma$ were $71 \%, 78 \%$, and $70 \%$, respectively. To differentiate the CD and NCGS groups from the control group, IL-15 had the highest sensitivity (82.70\%), specificity (56.50\%), positive predictive value (81.98\%), and negative predictive value $(57.78 \%)$, followed by IL-8 with the highest sensitivity of $74.50 \%$, specificity of $73.30 \%$, and positive and negative predictive values of $95.35 \%$ and $30.21 \%$, respectively. Conclusion: The obtained results demonstrate that IL-15 and IL-8 could be proposed as potential markers in their optimal cut-off points for distinguishing CD from the NCGS and the healthy control. Based on our findings, the evaluation of cytokine levels can be recommended as a useful tool for the diagnosis of CD and NCGS in a clinical practice. DOI: 10.29252/ibj.24.6.335
\end{abstract}

Keywords: Celiac disease, Cytokines, Sensitivity and specificity

Corresponding Author: Mohammad Rostami-Nejad

Gasteroentology and Liver Diseases Research Center, Research Institute for Gasteroenterology and Liver Diseases, Shahid Beheshti University of Medical Sciences, Tehran, Iran; Tel.: (+98-21) 224325259; E-mail: m.rostami@gmail.com

\section{INTRODUCTION}

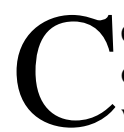
eliac disease is an autoimmune disorder occurred in genetically predisposed people whose small intestine is unable to properly absorb nutrients from food ${ }^{[1]}$. Gluten consumption by $\mathrm{CD}$ patients triggers an immune response in their small intestine. Gluten is a group of proteins, mainly found in wheat, rye, and barley, as well as in products derived from these grains. $\mathrm{CD}$ is a major public health concern all over the world and affects approximately $1 \%$ of the population of South America, Africa, Europe, and Asia $^{[2]}$. This rate varies from $0.5 \%$ to $1.0 \%$ for Iranian population $^{[3]}$. 
$\mathrm{CD}$ diagnosis is based on serological assays (endomysial antibody, deamidated gliadin peptide, and tTG), and patients with positive results are examined using endoscopy and small intestinal biopsies ${ }^{[4]}$. It is widely accepted that $\mathrm{CD}$ is a $\mathrm{T}$ cell-mediated disease that modifies gliadin-derived peptides represented by HLA-DQ2 and HLA-DQ8 ${ }^{[4]}$. The enzymatic activities of tTG can result in the activation of autoreactive T-helper cells, followed by the production of proinflammatory cytokines and intestinal inflammation $^{[5]}$.

Proinflammatory cytokines such as IFN- $\gamma$ and IL-15 play critical roles in CD pathogenesis as the elevated levels of these cytokines are related to the severity of the disease ${ }^{[5]}$. Meanwhile, production of IFN- $\gamma$ leads to $\mathrm{T}$ cells activation in small intestinal mucosa, which observed in the active form of $\mathrm{CD}^{[6]}$. Activation of intestinal intraepithelial lymphocytes is main cause of IL-15 elevation ${ }^{[7]}$. The role of proinflammatory cytokines such as IL- $1 \beta$ and TNF- $\alpha$ in mediating the mucosal damage has also been investigated. High amount of IL-1 $\beta$ and TNF- $\alpha$ results in overexpression of specific matrix metalloproteinases 1 and $3^{[5,8]}$. On the other hand, those subjects who are negative for CD and responded to the gluten-free diet are classified as having NCGS $S^{[9,10]}$. Diagnosis of NCGS remains as a controversial challenge, because there are no reliable diagnostic markers ${ }^{[11,12]}$. Since HLA-DQ2/DQ8 is present only in the minority of patients with NCGS, it has insufficient sensitivity for diagnosis of $\mathrm{NCGS}^{[13,14]}$. It has been reported that CD and NCGS patients have different reactions to gluten ingestion. CD patients have both innate and adaptive immune responses, but only innate immunity may be activated in NCGS patients ${ }^{[15]}$. Therefore, in both patient groups, the level of IL-1 $\beta$, IL- 6 , IL- 8 , IFN- $\gamma$, IL-2, and IL- 15 may be determining factors ${ }^{[16]}$.

At the systemic level, a few studies have measured the serum levels of cytokines, using ELISA for CD patients ${ }^{[5,17]}$. Findings have indicated that the elevated levels of IL2, IL-18, and IFN- $\gamma$ increase the chance of active $\mathrm{CD}^{[18-20]}$. As mentioned above, the inflammatory immune responses raised by the ingestion of gluten are involved in both CD and NCGS patients ${ }^{[15]}$; hence, it might be necessary to assess the diagnostic and predictive value of inflammatory cytokines as a tool for discrimination between CD and NCGS patients. The goal of the present study was comparing and evaluating the sensitivity, specificity, and predictive power of cytokines IL-6, IL-1, IL-15, IL8, and IFN- $\gamma$ by using AUC criteria in two subgroups, first by comparing CD with NCGS and then by comparing CD with healthy group.

\section{MATERIALS AND METHODS}

\section{Study population}

In January 2016, this study was conducted at the Research Institute for Gastroenterology and Liver Diseases at Shahid Beheshti University of Medical Sciences as the main referral center for CD patients in Tehran, Iran. The diagnosis of $\mathrm{CD}$ patients was performed based on the serological tests such as antitTG antibodies and endoscopy. Seropositive patients underwent endoscopy; however, with respect to the Marsh classification, those with the histopathological changes of small intestine were confirmed as CD patients. Serum samples were collected from $110 \mathrm{CD}$ patients. The gastrointestinal symptoms of patients were also collected immediately at the time of diagnosis. In addition, 46 subjects without any disease were included as control. Finally, 15 NCGS patients with negative serologic tests results for $\mathrm{CD}$ and wheat allergy were included in this study, but the patients with the history of other autoimmune disorders such as inflammatory bowel disease and Type 1 diabetes were excluded.

\section{Sample collection}

Peripheral blood samples $(10 \mathrm{ml})$ were obtained from each $\mathrm{CD}$ and NCGS patients and also healthy controls, and then serum was stored at $-80{ }^{\circ} \mathrm{C}$ immediately after taking the blood sample. Moreover, the characteristics and medical history of the participants were recorded by questionnaires.

\section{Measurement of cytokine levels}

The commercial ELISA kits (eBioscience, USA) were used to quantify the IL-1, IL-6, IL-8, IL-15 and IFN- $\gamma$ levels, according to the manufacturer's standard protocol. Serum or plasma samples $(50 \mu \mathrm{l})$ were used in these assays. The sensitivity and the brands of ELISA kits for IL-15, IL-8, IL-1, IL-6, and IFN- $\gamma$ were as $3.4 \mathrm{pg} / \mathrm{mL}$ (Invitrogen ${ }^{\mathrm{TM}}$ BMS2106), $2.0 \mathrm{pg} / \mathrm{mL} \quad$ (InvitrogenTM BMS2043TENCE), $0.3 \mathrm{pg} / \mathrm{mL}$ (Invitrogen ${ }^{\mathrm{TM}}$ BMS224-2), 0.01 $\mathrm{ng} / \mathrm{mL}$ (Invitrogen ${ }^{\mathrm{TM}} \mathrm{BMS} 214 \mathrm{TEN}$ ), and $0.99 \mathrm{pg} / \mathrm{mL}$ (Invitrogen $^{\mathrm{TM}} \mathrm{BMS} 228 \mathrm{CE}$ ). The sensitivity and specificity are statistical measures that refer to the ability of the test to correctly identify an individual as disease (CD) and disease-free (NCGS or control group), respectively. Positive predictive value and negative predictive value are the probabilities explaining that individuals with positive and negative clinical tests are truly disease and disease-free, respectively $^{[21]}$. 
Table 1. Description of cytokines in CD, NCGS, and control groups

\begin{tabular}{ccccc}
\hline Cytokines & Examined group & No. of patients & Mean $(\mathbf{n g} / \mathbf{m l})$ & SD \\
\hline \multirow{3}{*}{ IL-1 } & CD & 110 & 5.12 & 9.86 \\
& NCGS & 15 & 6.56 & 18.51 \\
& Control & 46 & 2.63 & 1.65 \\
IL-6 & CD & 110 & 9.79 & 7.11 \\
& NCGS & 15 & 8.38 & 5.90 \\
& Control & 46 & 11.17 & 10.18 \\
IL-8 & CD & 110 & 115.69 & 128.06 \\
& NCGS & 15 & 40.18 & 43.65 \\
& Control & 46 & 81.00 & 81.42 \\
IL-15 & CD & 110 & 219.57 & 370.14 \\
& NCGS & 15 & 96.33 & 54.37 \\
& Control & 46 & 143.57 & 226.43 \\
IFN- $\gamma$ & CD & 110 & 76.27 & 132.92 \\
& NCGS & 15 & 26.33 & 59.25 \\
& Control & 46 & 42.41 & 82.93 \\
\hline \multirow{3}{*}{} & & & &
\end{tabular}

\section{Statistical analysis}

In this study, ROC curve analysis and Youden's Index were employed to determine the optimal thresholds for detecting the high sensitivity, specificity, positive and negative predictive values of cytokines IL1 , IL-6, IL-8, IL-15, and IFN- $\gamma$. The mentioned cytokines were used as an accuracy diagnostic tool for distinguishing CD patients from NCGS and also from healthy groups. Moreover, the simultaneous effect of cytokines for the detection of $\mathrm{CD}$ patients was investigated using the logistic regression model. Statistical analyses were performed using IBM SPSS Statistics for Windows, Version 25.0. (IBM Corp., Armonk, NY, USA) and $R$ 3.4.1 software.

\section{Ethical statement}

The above-mentioned sampling protocols were approved by the Medical Ethics Committee of the
Research Institute for Gastroenterology and Liver Diseases, Shahid Beheshti University of Medical Sciences, Tehran, Tehran province, Iran (ethical code: IR.SBMU.RETECH.REC.1397.1013). Written informed consents were provided by the patients.

\section{RESULTS}

A total of 110 patients with $\mathrm{CD}$ (with the mean age of $33.6 \pm 11.87$ years old; $34.5 \%$ of them were male), 15 patients with NCGS (with the mean age of $38.7 \% \pm$ 13.75 years old; $46.7 \%$ of them were male), and 46 healthy individuals (with the mean age of $38.8 \pm 13.74$ years old; $43.4 \%$ of them were male) were enrolled in this study. The description of cytokines in each group is shown in Table 1. As shown in Table 2, the best cutoff points for the IL-1, IL-6, IL-8, IL-15, and IFN- $\gamma$ for

Table 2. ROC analysis of different cytokines for the diagnosis of CD in control and NCGS groups

\begin{tabular}{|c|c|c|c|c|c|c|c|}
\hline Group & Cytokines & $\begin{array}{c}\text { Cut-off point } \\
\text { (ng/ml) }\end{array}$ & $\mathbf{A U C}$ & Sensitivity & Specificity & PPV & NPV \\
\hline \multirow{6}{*}{$\mathrm{CD}$ vs. control } & IFN- $\gamma$ & 1.50 & 0.55 & 73.60 & 41.30 & 73.20 & 33.90 \\
\hline & IL-1 & 2.34 & 0.45 & 36.40 & 78.30 & 80.00 & 33.96 \\
\hline & IL-15 & 68.79 & 0.68 & 82.70 & 56.50 & 81.98 & 57.78 \\
\hline & IL-6 & 5.80 & 0.51 & 76.40 & 32.60 & 73.04 & 36.59 \\
\hline & IL-8 & 26.66 & 0.60 & 80.00 & 45.70 & 77.88 & 48.84 \\
\hline & Total $^{*}$ & 0.29 & 0.66 & 44.5 & 87.0 & 61.90 & 19.44 \\
\hline \multirow{6}{*}{ CD vs. NCGS } & IFN- $\gamma$ & 1.50 & 0.70 & 73.60 & 66.70 & 94.19 & 25.64 \\
\hline & IL-1 & 1.07 & 0.71 & 95.45 & 40.30 & 89.43 & 25.50 \\
\hline & IL-15 & 86.17 & 0.64 & 57.30 & 73.30 & 94.03 & 18.97 \\
\hline & IL-6 & 6.25 & 0.59 & 49.10 & 80.00 & 94.74 & 17.65 \\
\hline & IL-8 & 29.51 & 0.78 & 74.50 & 73.36 & 95.35 & 30.21 \\
\hline & Total & 0.13 & 0.75 & 70.2 & 60.0 & 76.60 & 11.22 \\
\hline
\end{tabular}

PPV, positive predictive value; NPV, negative predictive value 
the diagnosis of CD patients in the control group were $2.34 \mathrm{ng} / \mathrm{mL}, 5.80 \mathrm{ng} / \mathrm{mL}, 26.66 \mathrm{ng} / \mathrm{mL}, 68.79 \mathrm{ng} / \mathrm{mL}$, $1.50 \mathrm{ng} / \mathrm{mL}$, and for diagnosis of CD patients in NCGS group were $1.07 \mathrm{ng} / \mathrm{mL}, 6.25 \mathrm{ng} / \mathrm{mL}, 29.51 \mathrm{ng} / \mathrm{mL}$, $86.17 \mathrm{ng} / \mathrm{mL}$, and $1.50 \mathrm{ng} / \mathrm{ml}$, respectively. Besides, in the CD-NCGS group, none of the AUC values were
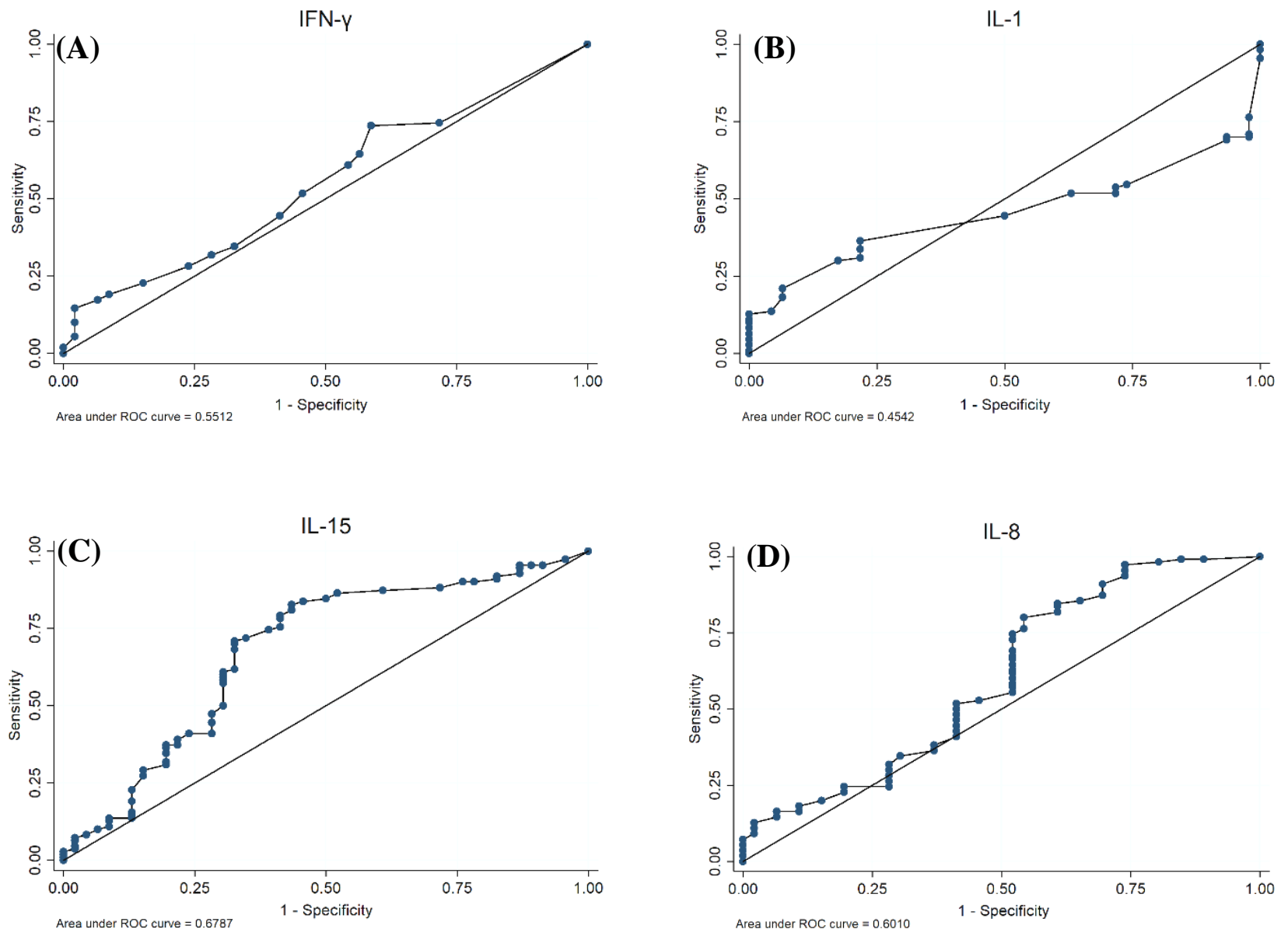

IL-6

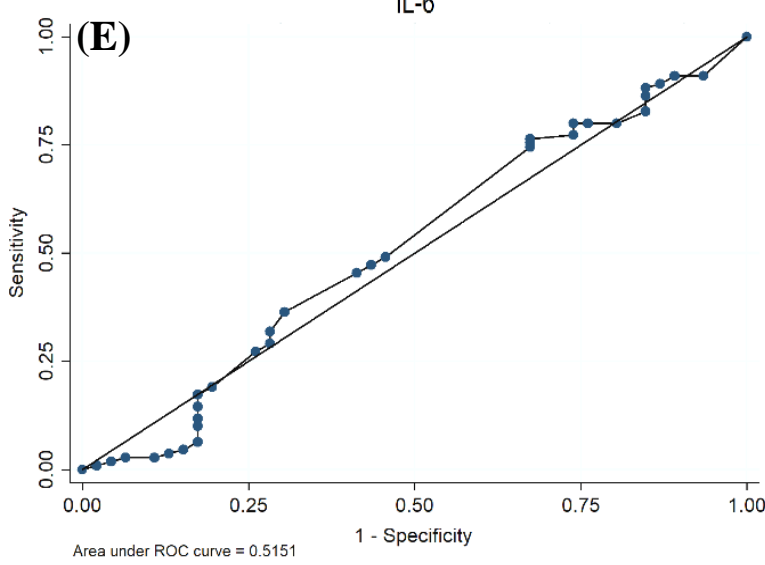

Fig. 1. ROC curve for CD patients and healthy controls showing the plot and the best cut-off point for (A) IFN- $\gamma$ (B) IL-1, (C) IL15, (D) IL-8, and (E) IL-6. 

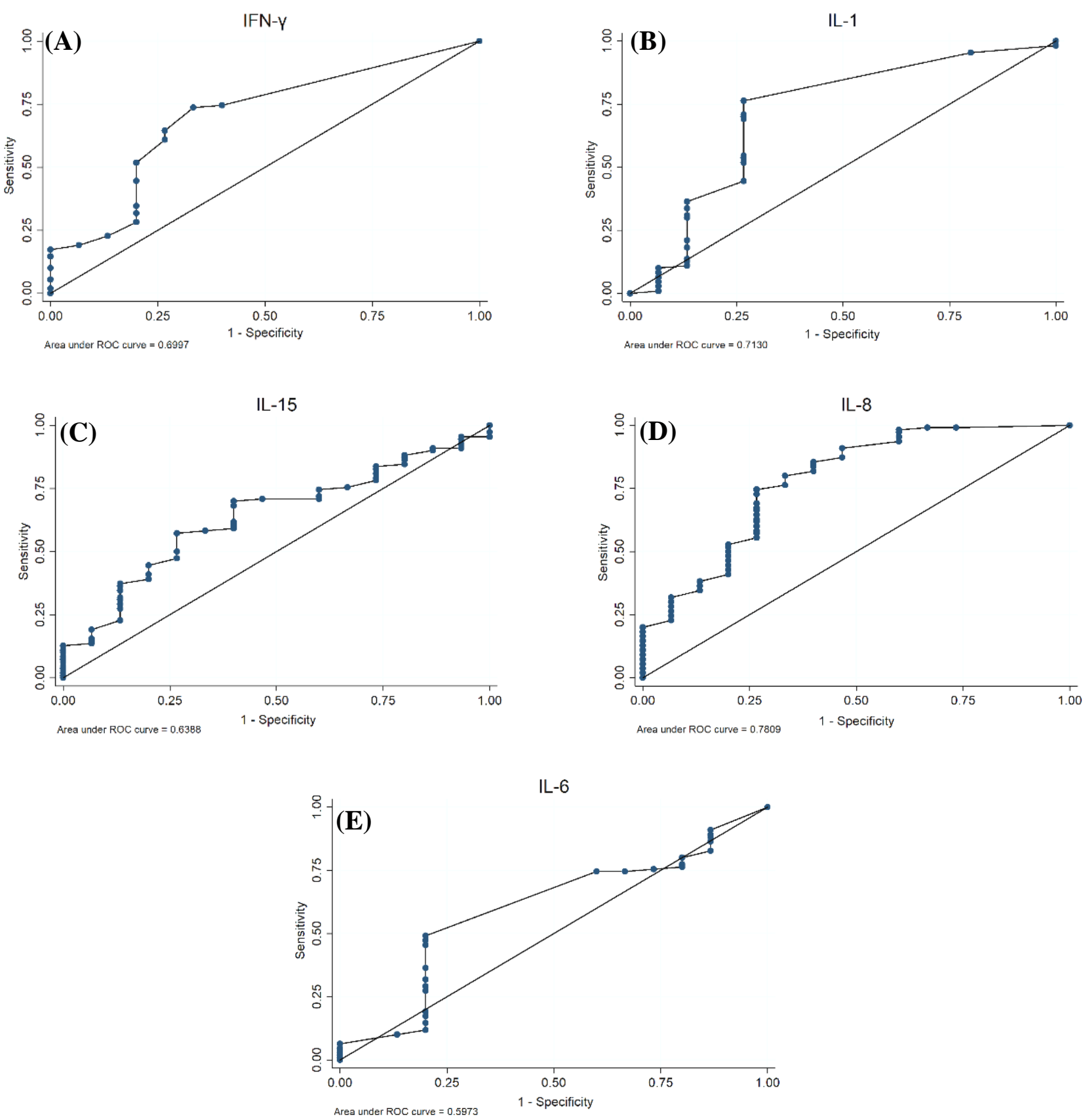

Fig. 2. ROC curve for CD patients and NCGS patients showing the plot and the best cut-off point for (A) IFN- $\gamma$ (B) IL-1, (C) IL-15, (D) IL-8, and (E) IL-6.

\section{DISCUSSION}

In this study, for the first time, the possible diagnostic performance of cytokines for CD and NCGS were evaluated. Our findings demonstrated that IL-8 and IL-15 had the highest sensitivities, specificities, positive and negative predictive values for the detection of the CD patients compared to the NCGS group and healthy control. Also, the combination of all investigated cytokines in the $\mathrm{CD}$ group indicated no significant effect on the diagnosis of CD patients. The pleiotropic cytokine IL-15, as a protein with a wide variety of biological functions, has 114 amino acids, which were primarily produced by mononuclear phagocytes in stressful conditions ${ }^{[22]}$. This cytokine plays a key role in the development of organ-specific autoimmune and inflammatory diseases ${ }^{[23]}$. Many studies have considered its performance on the pathogenesis of $\mathrm{CD}$ and also confirmed its effects on immunological responses ${ }^{[22]}$. In a study conducted by 
Di Sabatino et al. ${ }^{[24]}, 46 \mathrm{CD}$ patients and 22 healthy individuals were evaluated, and their results indicated that the expression of IL-15 level increased in the intestine of $\mathrm{CD}$ patients compared to the healthy group, which means that the lower immunological threshold of IL-15 in CD results in the development of other immune responses and small bowel lesion. Bernardo et $a l .{ }^{[25]}$ also obtained the same result after the evaluation of $42 \mathrm{CD}$ patients and 24 healthy individuals in a gastroenterology clinic for intestinal pathologies. However, IL-15 did not increase in NCGS group ${ }^{[7]}$. In our study, the diagnostic power of the IL-15 was evaluated and indicated that it is not an efficient tool for detecting CD (sensitivity $=57.30 \%$ ) in the NCGS group. Furthermore, more than $68.79 \mathrm{ng} / \mathrm{ml}$ of this cytokine could be considered as an evidence of CD by comparing it with the healthy control group. Heydari et al. $^{[7]}$ and Di Sabatini et al. ${ }^{[24]}$ reported that the serum IL- $1 \alpha$ and IL-1 $\beta$ levels were not significantly different among CD, NCGS, and healthy control groups. In contrast, Manavalan et al. ${ }^{[5]}$ showed a high level of IL$1 \beta$ in $\mathrm{CD}$ patients compared to the control group. Based on the different results of past studies, we examined the accuracy of this cytokine as a diagnostic tool for CD patients. Finally, it resulted in $95.45 \%$ sensitivity for the detection of CD patients against NCGS group, which makes it an appropriate tool for detecting CD.

IL-8, as a proinflammatory chemokine, belongs to chemokine family and plays a pivotal role in neutrophil degranulation. In previous studies, a significant expression of IL-8 was demonstrated in the CD patients compared to other groups, which could be explained as the long time activation of proinflammatory responses in the intestine of $\mathrm{CD}$ patients ${ }^{[7,26]}$. In addition, IL-8 (>29.5) almost had a high accuracy $(78 \%)$ to diagnose CD patients among NCGS population.

IL-6, a pleiotropic cytokine, is mainly produced in lamina propria myeloid cells in response to intestinal damage and has a significant function in inflammation, as well as in mediating the innate and adaptive immune responses, making IL-6 an important factor in CD pathogenesis ${ }^{[7,27]}$. The result of a number of previous studies showed the highest level of IL-6 in the serum of CD patients in comparison with the NCGS and control group ${ }^{[5,7,24]}$. In NCGS, the low level of IL-6 was reported, and its increased level was not sufficiently accurate for detecting CD patients in this study population ${ }^{[28]}$.

IFN- $\gamma$, an important mediator of immunity and inflammation, is expressed by Th1 cells. This critical cytokine plays an essential role in both innate and adaptive immune systems. Manavalan et al. ${ }^{[5]}$ reported an elevation in the levels of IFN- $\gamma$ in CD group. Many other studies have also shown that the increased expression level of IFN- $\gamma$ in lamina propria may lead to histological changes in the small bowel of $\mathrm{CD}$ patients $^{[5,29,30]}$. In a case-control study on 50 Iraqi CD patients, Abed et al. ${ }^{[30]}$ indicated a higher level of IFN$\gamma$ in CD group in comparison with control subjects. In our study, the increased production of IFN- $\gamma$ cytokine in active $\mathrm{CD}$ is in contrast with low levels of IFN- $\gamma$ in the NCGS group. Di Sabatino et al. ${ }^{[24]}$ and Losurdo et $a l .{ }^{[31]}$ have also shown that the expression of IFN- $\gamma$ in CD was significantly higher than both control and NCGS subjects. These results are completely consistent with our research results. As in our study, when the level of IFN- $\gamma$ is higher than $1.50 \mathrm{ng} / \mathrm{ml}$, it has an acceptable accuracy and can be used as a tool to detect CD patients among NCGS group. These results are completely consistent with our research results.

This study indicated that the serum levels of IL-8 and IL-15 in their best cut-off points were potential biomarkers for distinguish CD patients from NCGS and control groups. Moreover, the simultaneous increment of these five cytokines (IL-15, IL-8, IL-1, IL-6, and IFN- $\gamma$ ) provided $75 \%$ diagnostic accuracy in distinguishing the NCGS groups from CD patients. Although these tests cannot be used for measuring the systemic inflammation at this time, our findings propose the use of cytokines assay for the diagnosis of $\mathrm{CD}$ and NCGS in a clinical practice.

CONFLICT OF INTEREST. None declared.

\section{REFERENCE}

1. Rodrigo L, Perez-Martinez I, Lauret-Braña E, SuarezGonzalez A. Evaluation of efficacy and adherence to gluten-free diet in patients with celiac disease. Nutrients 2018; 10: 1777.

2. Kelly CP, Bai JC, Liu E, Leffler DA. Advances in diagnosis and management of celiac disease. Gastroenterology 2015; 148(6): 1175-1186.

3. Ahadi Z, Shafiee G, Razmandeh R, Keshtkar AA, Najafi sani M, Azemati B, Sanaei M, Heshmat R. Prevalence of celiac disease among the Iranian population:A systematic review and meta-analysis of observational studies. Turkish journal of gastroenterology 2016; 27(2): 122-128.

4. Kaswala D, Veeraraghavan G, Kelly C, Leffler DJD. Celiac disease: diagnostic standards and dilemmas. Diseases 2015; 3(2): 86-101.

5. Manavalan JS, Hernandez L, Shah JG, Konikkara J, Najver AJ, Lee AR, Ciaccio E, Minaya MT, Green PH, Bhagat G. Serum cytokine elevations in celiac disease: association with disease presentation. Human immunology 2010; 71(1): 50-57. 
6. Schuppan D, Junker Y, Barisani D. Celiac disease: from pathogenesis to novel therapies. Gastroenterology 2009; 137(6): 1912-1933.

7. Heydari F, Rostami-Nejad M, Moheb-Alian A, Mollahoseini MH, Rostami K, Aghamohammadi E, Zali MR. Serum cytokines profile in treated celiac disease compared with non-celiac gluten sensitivity and control: a marker for differention. Journal of gastrointestinal and liver diseases 2018; 27(3): 241-247.

8. Daum S, Bauer U, Foss H, Schuppan D, Stein H, Riecken Eo, Ullrich R. Increased expression of mRNA for matrix metalloproteinases-1 and-3 and tissue inhibitor of metalloproteinases-1 in intestinal biopsy specimens from patients with coeliac disease. Gut 1999; 44(1): 17-25.

9. Mansueto P, Seidita A, D'Alcamo A, Carroccio A. Nonceliac gluten sensitivity: literature review. Journal of the American college of nutrition 2014; 33(1): 39-54.

10. Lionetti E, Leonardi S, Franzonello C, Mancardi M, Ruggieri M, Catassi CJN. Gluten psychosis. Nutrients 2015; 7(7):5532-5539.

11. Biesiekierski JR, Iven J. Non-coeliac gluten sensitivity. United European gastroenterology journal 2015; 3(2): 160-165.

12. Molina-Infante J, Santolaria S, Sanders DS, FernándezBañares F, Systematic review: noncoeliac gluten sensitivity. Alimentary pharmacology and theraputics 2015; 41(9): 807-820.

13. Castro-Antunes MM, Crovella S, Brandão LAC, Guimaraes RL, Motta MEFA, Silva GA. Fequency distribution of HLA DQ2 and DQ8 in celiac patients and first-degree relatives in recife, northeastern Brazil. Clinics (Sao Paulo) 2011; 66(2): 227-231.

14. Czaja-Bulsa G. Non coeliac gluten sensitivity-A new disease with gluten intolerance. Clinical nutrition 2015; 34(2): 189-194.

15. Peña AS. Immunogenetics of non celiac gluten sensitivity. Gastroenterology and hepatology from bed to bench 2014; 7(1): 1-5.

16. Vojdani A, Perlmutter D. Differentiation between celiac disease, nonceliac gluten sensitivity, and their overlapping with Crohn's disease. Case reports in immunology 2013; 248482.

17. Iftikhar $\mathrm{R}$, Jamal $\mathrm{S}$, Zafar A, Saadia A. Histopathological and immunohistochemical analysis of small intestinal biopsies in adults suspected of celiac disease. Journal of the college of physicians and surgeons-Pakistan 2016; 26(10): 827-830.

18. Penedo-Pita M, Peteiro-Cartelle J, nutrition. Increased serum levels of interleukin-2 and soluble interleukin-2 receptor in celiac disease. Journal of pediatric gastroenterology and nutrition 1991; 12(1): 56-60.

19. Blanco A, Garrote J, Arranz E, Alonso M, Clavo C, nutrition. Increased serum IL-2R levels in coeliac disease are related to CD4 but not CD8 antigens. Journal of pediatric gastroenterology and nutrition 1992; 15(4): 413-417.

20. Cataldo F, Marino V, nutrition. Increased prevalence of autoimmune diseases in first-degree relatives of patients with celiac disease. Journal of pediatric gastroenterology and nutrition 2003; 36(4): 470-473.

21. Parikh R, Mathai A, Parikh S, Sekhar G, Thomas R. Understanding and using sensitivity, specificity and predictive values. Indian journal of ophthalmology 2008; 56(1): 45-50.

22. Abadie V, Jabri BJ. IL-15: a central regulator of celiac disease immunopathology. Immunological reviews 2014; 260(1): 221-234.

23. Yu H, Sui Y, Wang Y,Sato N, Frey B, Xia Z, Waldmann TA, Berzofsky J. Interleukin-15 constrains mucosal $\mathrm{T}$ helper 17 cell generation: Influence of mononuclear phagocytes. PLoS one 2015; 10(11): e0143001.

24. Di Sabatino A, Ciccocioppo R, Cupelli F, Cinque B, D Millimaggi M M Clarkson, M Paulli, M G Cifone, and G R Corazza. Epithelium derived interleukin 15 regulates intraepithelial lymphocyte Th1 cytokine production, cytotoxicity, and survival in coeliac disease. Gut 2006; 55(4): 469-477.

25. Bernardo D, Garrote JA, Allegretti Y,Leon A, Gomez E, Bermejeo JF, Calvo C, Riestra S, Fernandez S, Blanco A, Chirdo F, Arranz E. Higher constitutive IL15R $\alpha$ expression and lower IL-15 response threshold in coeliac disease patients. Clinical and experimental immunology 2008; 154(1): 64-73.

26. David J, Dominguez C, Hamilton D, Palena C. The IL8/IL-8R axis: a double agent in tumor immune resistance. Vaccines 2016; 4(3): 22.

27. Dema B, Martinez A, Fernandez-Arquero M, Maluenda C, Polancol L, Flqueredo MA, Concha EG, Urcelay E, Nunez C. The IL6-174G/C polymorphism is associated with celiac disease susceptibility in girls. Human immunology 2009; 70(3): 191-194.

28. Pone A, Lammers KM, Casolaro V, Cammarota M, Giuliano MT, De Rosa M, Stefanile R, Mazzarella G, Tolone C, Russo MI, Esposito P, Ferraraccio F, Cartenì M, Riegler G, de Magistris L, Fasano A. Divergence of gut permeability and mucosal immune gene expression in two gluten-associated conditions. BMC medicine 2011; 9(1): 9-23.

29. Ferber IA, Brocke S, Taylor-Edwards C, Ridgway W, Dinisco C, Steinman L, Dalton D, Fathman CG. Mice with a disrupted IFN-gamma gene are susceptible to the induction of experimental autoimmune encephalomyelitis (EAE). The journal of immunology 1996; 156(1): 5-7

30. Abed RM, Yaaqoob LA, Alwan IA, Samir HF, Jasim RA. Evaluation of some serum cytokine and adipokines profile in Iraqi celiac patients before and after treatment. Journal of pharmacy and biological sciences 2017; 12(6): 36-41.

31. Losurdo G, Giorgio F, Piscitelli D,Montenegro L, Covelli C, Flore MG, Giangaspero A, Lannone A, Principi M, Amoruso A, Barone M, Di Leo A, Leradi E. May the assessment of baseline mucosal molecular pattern predict the development of gluten related disorders among microscopic enteritis. World journal of gastroenterology 2016; 22(35): 8017-8025. 